\title{
ARTICLE
}

\section{Imparting amphiphobicity on single-crystalline porous materials}

Qi Sun ${ }^{1,2}$, Hongming He${ }^{2}$, Wen-Yang Gao², Briana Aguila², Lukasz Wojtas², Zhifeng Dai', Jixue Li ${ }^{3}$, Yu-Sheng Chen ${ }^{4}$, Feng-Shou Xiao ${ }^{1} \&$ Shengqian $\mathrm{Ma}^{2}$

The sophisticated control of surface wettability for target-specific applications has attracted widespread interest for use in a plethora of applications. Despite the recent advances in modification of non-porous materials, surface wettability control of porous materials, particularly single crystalline, remains undeveloped. Here we contribute a general method to impart amphiphobicity on single-crystalline porous materials as demonstrated by chemically coating the exterior of metal-organic framework (MOF) crystals with an amphiphobic surface. As amphiphobic porous materials, the resultant MOF crystals exhibit both superhydrophobicity and oleophobicity in addition to retaining high crystallinity and intact porosity. The chemical shielding effect resulting from the amphiphobicity of the MOFs is illustrated by their performances in water/organic vapour adsorption, as well as long-term ultrastability under highly humidified $\mathrm{CO}_{2}$ environments and exceptional chemical stability in acid/base aqueous solutions. Our work thereby pioneers a perspective to protect crystalline porous materials under various chemical environments for numerous applications.

\footnotetext{
${ }^{1}$ Key Lab of Applied Chemistry of Zhejiang Province and Department of Chemistry, Zhejiang University, Hangzhou 310028, China. ${ }^{2}$ Department of Chemistry, University of South Florida, 4202 East Fowler Avenue, Tampa, Florida 33620, USA. ${ }^{3}$ Electron Microscopy Centre, Zhejiang University, Hangzhou 310027, China. ${ }^{4}$ ChemMatCARS, Center for Advanced Radiation Sources, The University of Chicago, 9700 South Cass Avenue, Argonne, Illinois 60439, USA. Correspondence and requests for materials should be addressed to F.-S.X. (email: fsxiao@zju.edu.cn) or to S.M. (email: sqma@usf.edu).
} 
T he custom design of surfaces with controlled wettability properties has been attracting continuous attention from both academia and industry ${ }^{1-7}$. In particular, amphiphobic surfaces ${ }^{8-12}$ that repel water and compounds with low surface tension (for example, oils) are of great interest due to their various prospective applications, such as oil transportation ${ }^{13}$, microfluidics ${ }^{14}$ and nano-object manipulation ${ }^{15}$. Albeit tremendous efforts have been devoted to creating such amphiphobic surfaces on a variety of substrates over the past several years ${ }^{8-12}$, the impartment of amphiphobicity on porous materials, specifically single crystalline, has not yet been explored.

As an emerging class of porous materials with high single crystallinity, metal-organic frameworks $(\mathrm{MOFs})^{16-18}$, which feature amenability to design, high surface areas, tunable pore sizes and tailorable functionality, have recently been extensively investigated for applications in gas storage ${ }^{99,20}$, separation ${ }^{11,22}$, carbon capture $^{23,24}$, catalysis ${ }^{25-28}$, sensing ${ }^{29,30}$ and so on (refs $31-34$ ). However, an issue for their wide applications in practice includes the performance under a variety of environments (for example, stability in humid conditions, interferences by organic vapours in the atmosphere ${ }^{35-38}$, which necessitate the sophisticated control of the surface wettability of MOFs. Recent studies indicate the observation of framework degradation on the exposure of MOFs to wet $\mathrm{CO}_{2}$, even for those claimed with moisture stability ${ }^{39,40}$. To repel water molecules, thereby protecting MOFs against hydrolysis ${ }^{41-53}$ while preserving crystallinity and intact porosity, some approaches have been developed to impart hydrophobicity/ superhydrophobicity on the exterior surfaces of MOFs. For example, Jiang and colleagues ${ }^{54}$ recently developed a facile yet general coating approach to modify hydrophobic polydimethysiloxane on the surfaces of MOF materials, which exhibited significant enhancement in moisture/water stability. However, some environments coexist with water and organic compounds. Therefore, amphiphobicity is needed to repel both water and organic molecules, to prevent the water attack while minimizing the interferences by organic compounds. Nonetheless, amphiphobic MOFs have not yet been achieved, despite their intriguing properties and attractive potentials for a variety of applications ${ }^{13-15}$.

To impart amphiphobicity on the MOFs, in this contribution we rationally designed vinyl-functionalized linkers for targeted construction of MOFs, where the vinyl groups can remain intact during the formation process of MOF crystals, yet are sufficiently reactive for further chemical modifications ${ }^{55}$. After controllable functionalization of the exterior amphiphilic crystal surfaces with perfluoroalkyl groups via thiol-ene reaction, the resultant MOFs exhibit both superhydrophobicity and oleophobicity, while retaining high crystallinity and intact porosity.

\section{Results}

Synthesis of vinyl prefunctionalized MOF. As a representative example of MOFs, a vinyl-functionalized crystalline zeolite imidazole framework was prepared using the protocol reported in the literature ${ }^{56}$ and it is isostructural with ZIF-8 as revealed by single-crystal X-ray diffraction (XRD) analysis (Supplementary Fig. 1). The guest solvent-free crystal product is designated as ZIF-8-V with a formula of $\mathrm{Zn}\left(\mathrm{C}_{5} \mathrm{H}_{5} \mathrm{~N}_{2}\right)_{2}$ and the retention of vinyl groups on the linkers during the crystal formation process was further confirmed by liquid ${ }^{1} \mathrm{H}$ NMR analysis of the digested ZIF-8-V sample (Supplementary Figs 2 and 3).

Crystal surface coating with perfluoroalkyl groups. To controllably introduce the fluorinated groups onto the exterior surface of ZIF-8-V, we hypothesize that, if a relatively bulky fluorinated compound that cannot permeate into the pore of the material is employed, the reaction would only occur on the exterior surface of the crystals. In addition, if these fluorocarbon chains are long enough, the surface energy of the sample would be significantly reduced ${ }^{9,10}$ (Fig. 1). To demonstrate this proof-ofconcept, $1 \mathrm{H}, 1 \mathrm{H}, 2 \mathrm{H}, 2 \mathrm{H}$-perfluorodecanethiol was chosen as a typical compound for the surface modification of ZIF-8-V crystals, which was achieved via the thiol-ene click reaction ${ }^{57}$ to afford ZIF-8-VF (Fig. 2).

Structural characterization. Figure 3a shows powder XRD (PXRD) patterns of ZIF-8-V and ZIF-8-VF, which agree well with the calculated ones of ZIF-8-V, indicating the good retention of crystallinity and structural integrity during the post-synthetic modification process. $\mathrm{N}_{2}$ sorption isotherms collected at $77 \mathrm{~K}$ (Fig. 3b) reveal that both ZIF-8-V and ZIF-8-VF exhibit the classic type I adsorption behaviour, a characteristic of microporous materials. Derived from the $\mathrm{N}_{2}$ adsorption data, ZIF-8-V and ZIF-8-VF have similar Brunauer-Emmett-Teller (BET) surface areas (816 and $850 \mathrm{~m}^{2} \mathrm{~g}^{-1}$ ) and pore volumes $\left(0.42 \mathrm{~cm}^{3} \mathrm{~g}^{-1}\right)$, suggesting that the postmodification process should primarily occur on the exterior surface of the crystals with negligible blockage of the pores in the pristine material, thereby still accessible for guest gas molecules. The scanning electron microscopy (SEM) images (Fig. 3c,d) show that there is little change in the overall morphology of the crystals after the chemical modification reaction but the surfaces of the ZIF-8-VF crystals are rougher than those of ZIF-8-V crystals, further indicating the occurrence of the reaction on the exterior surface of the crystals. It is worth noting that such roughness of the surface has been reported to be beneficial to the enhancement of the hydrophobicity and oleophobicity ${ }^{8}$.

To identify the surface coating, the ${ }^{13} \mathrm{C}$ and ${ }^{19} \mathrm{~F}$ MAS NMR spectra of ZIF-8-V and ZIF-8-VF were collected (Fig. 3e and Supplementary Fig. 4). Albeit no distinct difference in the ${ }^{13} \mathrm{C}$ NMR spectra are observed for the two samples, ZIF-8-VF shows clear ${ }^{19} \mathrm{~F}$ NMR signals with the same chemical shifts as those of the $1 \mathrm{H}, 1 \mathrm{H}, 2 \mathrm{H}, 2 \mathrm{H}$-perfluorodecanethiol compound (Supplementary Fig. 5), which can be attributed to the much higher natural abundance of ${ }^{19} \mathrm{~F}$ nuclei than that of ${ }^{13} \mathrm{C}$ nuclei. In addition, the Fourier transform infrared spectra of the ZIF-8-VF show the characteristic bands of C-F at 1,241 and $1,211 \mathrm{~cm}^{-1}$ as compared with the pristine ZIF-8-V (Supplementary Fig. 6). These results indicate that the perfluoroalkyl groups have been successfully introduced on ZIF-8-V, but the grafting degree is relatively low. To quantify the degree of postsynthetic modification, the ZIF-8-VF crystals were digested and analysed by the liquid ${ }^{1} \mathrm{H}$ NMR spectroscopy (Supplementary Fig. 7). The results show that $98 \%$ of vinyl groups are still intact, which means that $\sim 2 \%$ of the vinyl groups are involved in the thiol-ene reaction

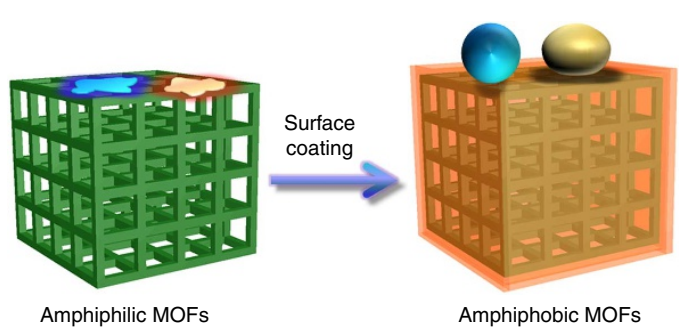

Figure 1 | Amphiphobic surface engineering for MOFs. The resultant MOFs exhibit both superhydrophobicity and oleophobicity, while retaining high crystallinity and intact porosity. 


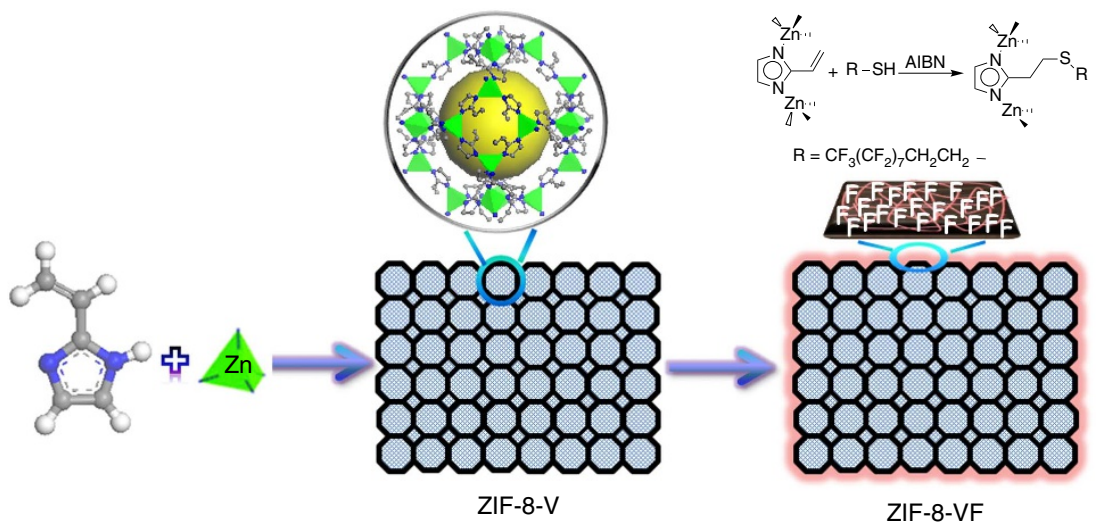

Figure 2 | Schematic illustration to impart amphiphobicity on ZIF-8-V. Synthetic route to create amphiphobic surface via grafting perfluoroalkyl groups on the exterior surface of the ZIF-8-V crystal.
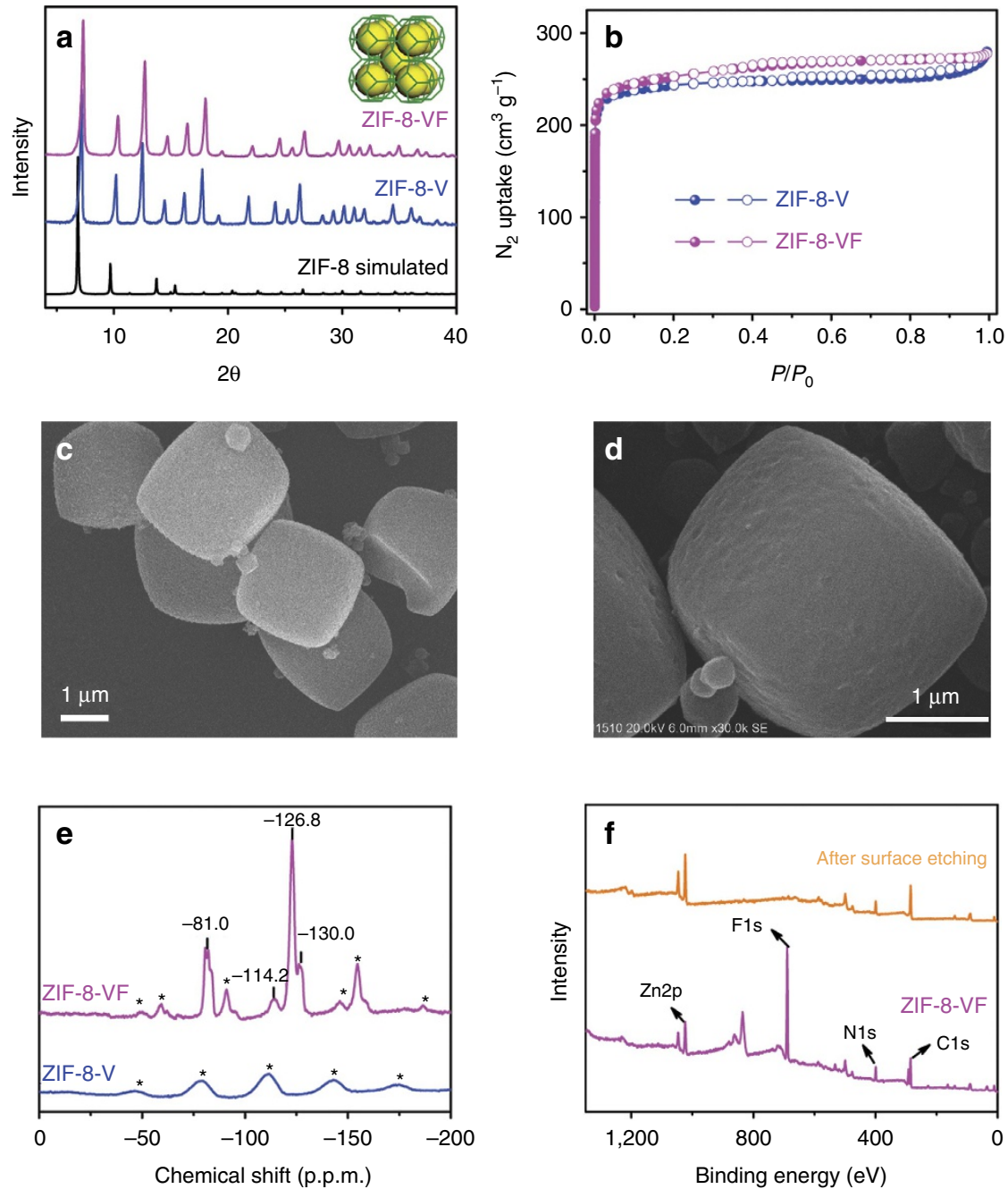

Figure 3 | Structural characterization. (a) PXRD patterns, (b) $\mathrm{N}_{2}$ sorption isotherms measured at $77 \mathrm{~K}$, (c) SEM image of ZIF-8-V, (d) SEM image of ZIF-8-VF, (e) ${ }^{19} \mathrm{~F}$ MAS NMR curves and (f) X-ray photoemission spectra of ZIF-8-VF before and after surface $\mathrm{Ar}^{+}{ }^{+}$ions etching ( ${ }^{*}$ side band).

probably occurring only on the exterior surface of the ZIF-8-V. To further prove the exterior surface modification, X-ray photoemission spectroscopy experiments were conducted (Fig. 3f and Supplementary Fig. 8), which reveal that ZIF-8-VF exhibits very strong signals associated with the $\mathrm{F}$ species. However, after the surface removal by $\mathrm{Ar}^{+}$ion-etching treatment $(2 \mathrm{KeV}, 100 \mathrm{~s})$, the amount of residual F species is very small. These results suggest that the perfluoroalkyl groups should be mainly attached to the vinyl groups on the exterior surface of ZIF-8-V crystals, which is further confirmed by the energy-dispersive X-ray mapping of the ZIF-8-VF sample before and after surface plasma cleaning process (Supplementary Figs 9 and 10). 
a
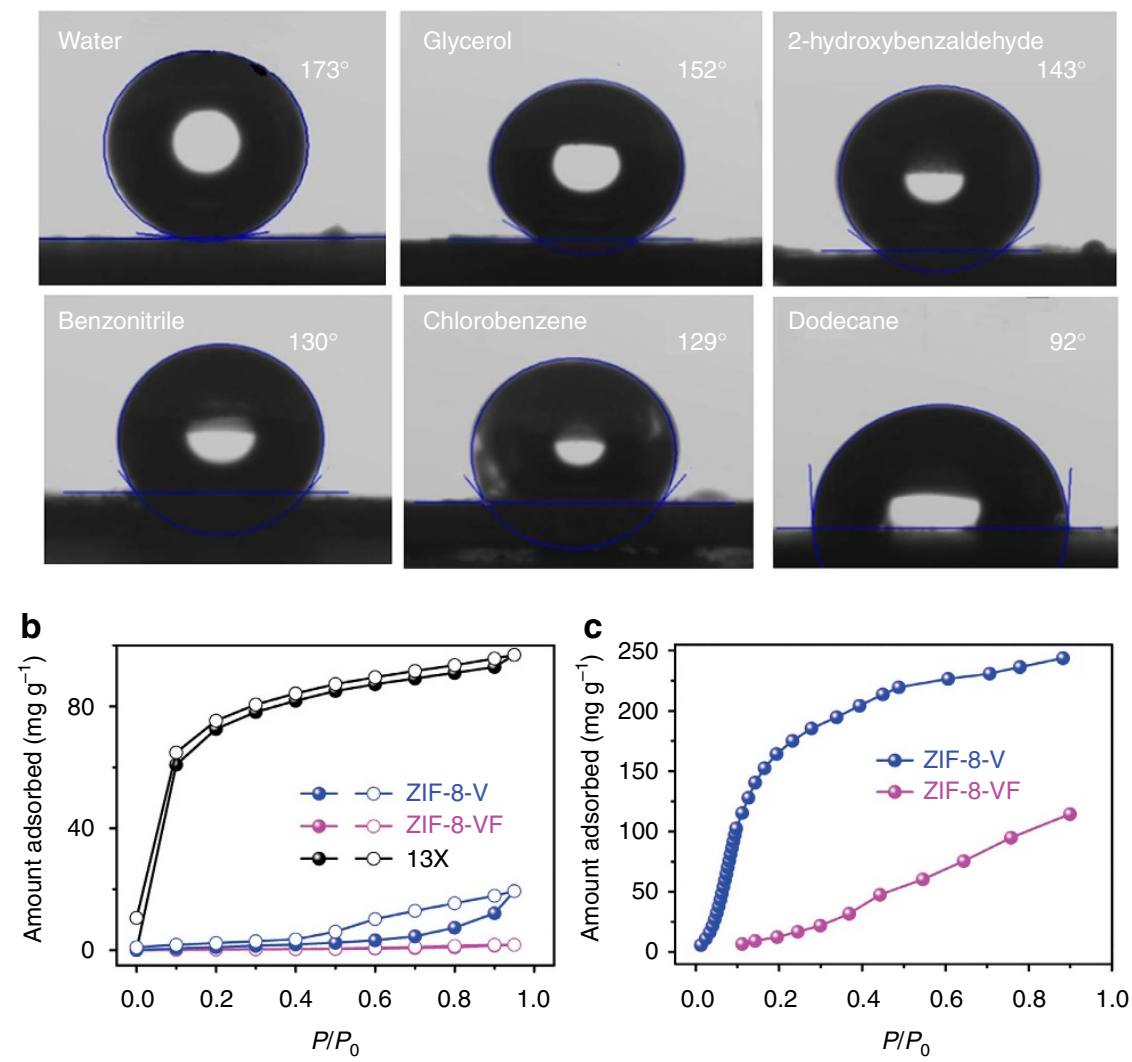

Figure 4 | Wettability tests and vapour sorption performance. (a) Contact angles of various liquid on the pressed pellet made using ZIF-8-VF sample, (b) water adsorption (solid symbols) and desorption (open symbols) isotherms collected at $298 \mathrm{~K}$ and (c) toluene adsorption isotherms collected at $298 \mathrm{~K}$.

Examination of amphiphobic properties. The surface wettability of ZIF-8-V and ZIF-8-VF was characterized by contact-angle measurements. Figure $4 \mathrm{a}$ shows contact angles of water and a series of organic compounds on the surface of ZIF-8-VF. Notably, the contact angle of water on ZIF-8-VF sample is as high as $173^{\circ}$, indicating its extraordinarily superhydrophobic feature. In contrast, ZIF-8-V gives the water contact angle at $89^{\circ}$ (Supplementary Fig. 11). These results confirm that the surface coating of perfluoroalkyl groups significantly enhances the hydrophobicity of the material. Furthermore, when a series of organic compounds with different surface tensions, including glycerol, 2-hydroxybenzaldehyde, benzonitrile, chlorobenzene and dodecane, were contacted with the surface of ZIF-8-VF, the contact angles at $150^{\circ}, 143^{\circ}, 130^{\circ}, 129^{\circ}$ and $92^{\circ}$, respectively, were observed, indicating the oleophobic feature of ZIF-8-V. The results of the contact-angle experiments coupled with the permanent porosity, as revealed from the $\mathrm{N}_{2}$ sorption measurement, thereby identify ZIF-8-VF as an amphiphobic porous material. In contrast, the contact angles of 2-hydroxybenzaldehyde, benzonitrile, chlorobenzene and dodecane on ZIF-8-V are $<5^{\circ}$ (Supplementary Fig. 12), indicating its superoleophilic nature. The superhydrophobic behaviour of the ZIF-8-VF was further illustrated by water vapour adsorption experiments (Fig. 4b). As a comparison, hydrophilic zeolite $13 \times$ exhibits strong affinity for water even at very low relative humidity $\left(P / P_{0}<0.1\right)$. Hydrophobic ZIF-8-V exhibits a hysteresis loop at relative humidity $>0.5$, indicating that ZIF- $8-\mathrm{V}$ is capable of adsorbing water at high humidity. Interestingly, ZIF-8-VF adsorbs a negligible amount of water even at $P / P_{0}$ up to 0.9 , which is anticipated to be effective to preclude the entrance of water within the interior pores of the $\mathrm{MOF}^{58-60}$. Toluene sorption isotherms collected at $298 \mathrm{~K}$ also reveal quite different adsorption behaviours for ZIF-8-V and ZIF-8-VF (Fig. 4c). For example, when $P / P_{0}$ is 0.15 , it is observed that the adsorption capacity of toluene in ZIF-8-V and ZIF-8-VF is 143 and $7 \mathrm{mgg}^{-1}$, respectively. The repellency of ZIF-8-VF for toluene should stem from its excellent oleophobicity. The above results highlight that the surface-coated amphiphobic perfluoroalkyl groups serve as a shield to effectively prevent the access of water and organic compounds into the micropores of MOFs.

Investigation of chemical shielding effect. The long-term stability under practical environments (for example, humidity) has been recognized as an issue for MOFs, which can be accessed via the method of accelerated ageing ${ }^{39}$. To evaluate the chemical shielding effect resulting from amphiphobicity, the PXRD patterns of various ZIF materials that are exposed to $100 \%$ relative humidity under $\mathrm{CO}_{2}$ atmosphere at $45^{\circ} \mathrm{C}$ were monitored for different duration times. When ZIF-8, one of the claimed very stable MOF materials ${ }^{61,62}$, was aged under the above conditions for $16 \mathrm{~h}$, it was observed that some additional peaks associated to an unknown phase appeared, as shown by the black arrows in Fig. 5a. Moreover, the ratio of the unknown phase increases with the increase of exposure time. For instance, after $240 \mathrm{~h}$, a large portion of ZIF- 8 was transformed, indicative of structural degradation of ZIF-8. This conclusion is supported by $\mathrm{N}_{2}$ sorption studies at $77 \mathrm{~K}$, suggesting a remarkable reduction in the surface area (from 957 to $378 \mathrm{~m}^{2} \mathrm{~g}^{-1}$; Supplementary Fig. 13), as well as SEM images showing clear cracks of the crystals (Fig. 5b,c). Similar changes in the PXRD patterns were also observed for ZIF-8-V (Supplementary Fig. 14). In striking contrast, ZIF-8-VF does not experience any change in the PXRD patterns, even after ageing under the above conditions over $720 \mathrm{~h}$. 

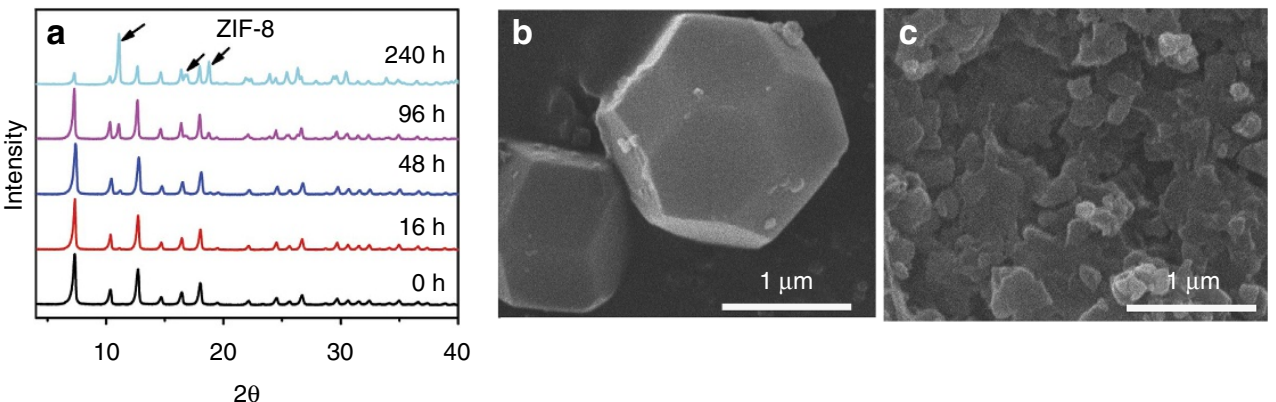

$2 \theta$
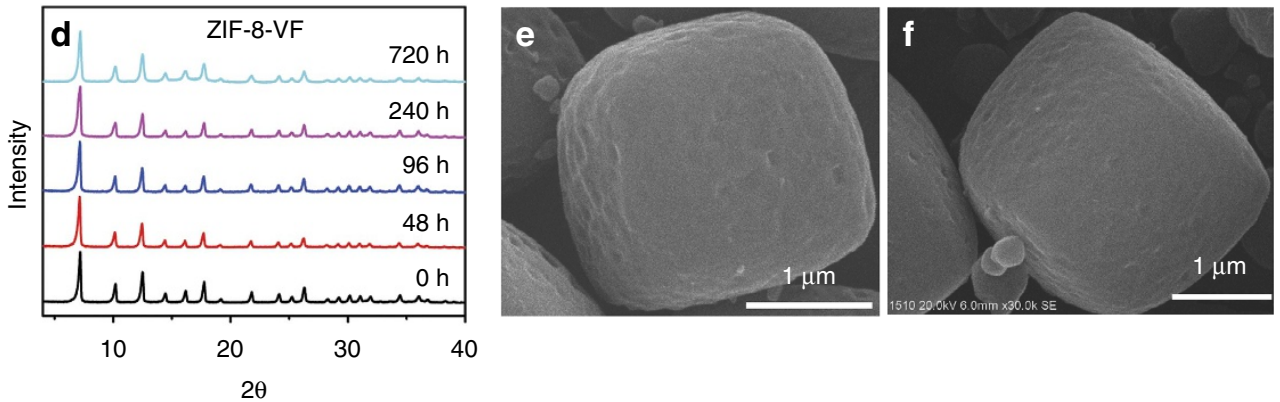

Figure 5 | Stability comparison of ZIF-8 and ZIF-8-VF. (a,d) Selected PXRD patterns of ZIF-8 and ZIF-8-VF ageing under 1 atm of water saturated $\mathrm{CO}_{2}$ at $45^{\circ} \mathrm{C}$ for different duration times. (b,c) SEM images of ZIF-8 before and after ageing under the above conditions for $240 \mathrm{~h}$. (e,f) SEM images of ZIF-8-VF before and after aging under the above conditions for $720 \mathrm{~h}$. Main peaks of unknown crystalline phase are marked with black arrows.
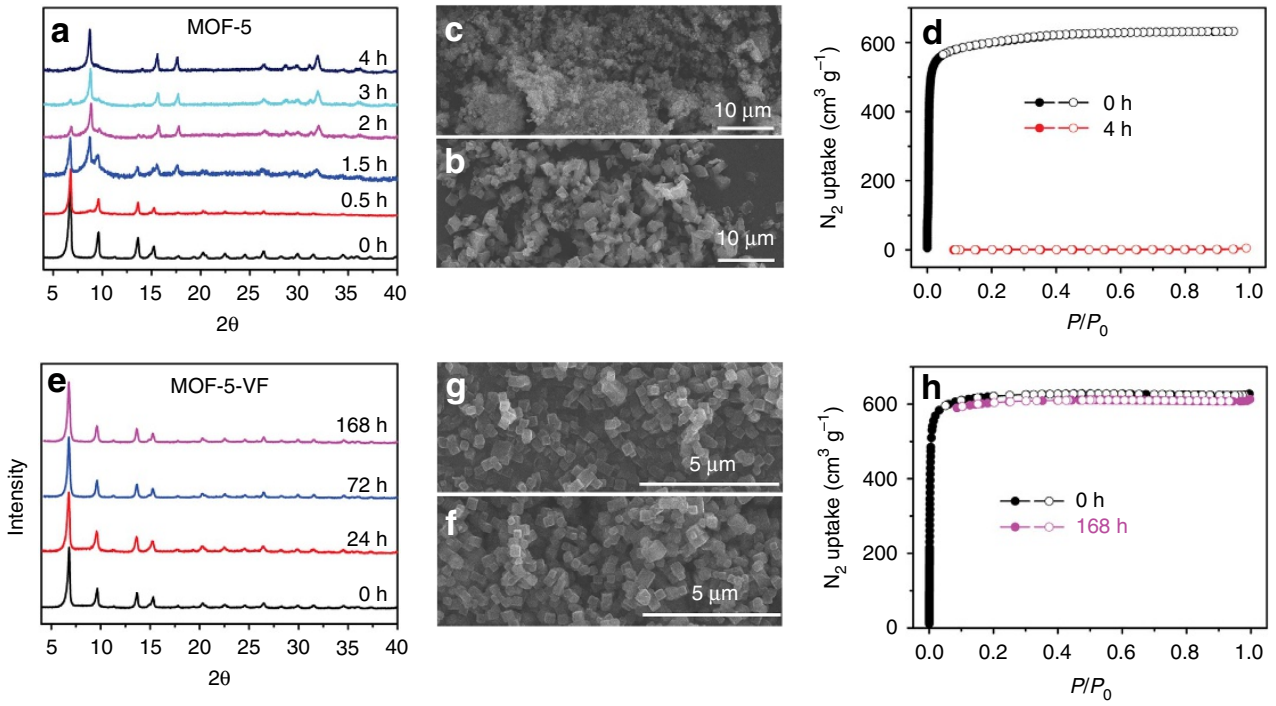

Figure 6 | Stability comparison of MOF-5 and MOF-5-VF. (a,e) Selected PXRD patterns of MOF-5 and MOF-5-VF ageing under 1 atm of water saturated $\mathrm{CO}_{2}$ at $45^{\circ} \mathrm{C}$ for different duration times. (b,c) SEM images and (d) $\mathrm{N}_{2}$ sorption isotherms of MOF- 5 before and after ageing under the above conditions for $4 \mathrm{~h}$. (f,g) SEM images and (h) $\mathrm{N}_{2}$ sorption isotherms of MOF-5-VF before and after aging under the above conditions for $168 \mathrm{~h}$.

The SEM images show that the ZIF-8-VF sample has maintained perfect crystal morphology and its surface area is also fully retained $\left(802 \mathrm{~m}^{2} \mathrm{~g}^{-1}\right.$; Supplementary Fig. 15). These results highlight that the amphiphobic surface can serve as a chemical shield to effectively prevent ZIF-8-VF from being attacked by the mixture of $\mathrm{H}_{2} \mathrm{O}$ and $\mathrm{CO}_{2}$.

\section{Discussion}

To demonstrate the general applicability of the strategy presented herein, vinyl-functionalized MOF (MOF-5-V; Supplementary Fig. 16) isostructural with MOF-5, which is notoriously water/ moisture unstable, was synthesized using the custom-designed ligand of 2-vinylterephthalic acid (Supplementary Fig. 17). After surface chemical coating of the perfluoroalkyl groups (Supplementary Figs 18-22), the resultant material (MOF-5-VF) demonstrates amphiphobic property (Supplementary Figs 23 and 24), which renders it with extraordinary tolerance to the humidified $\mathrm{CO}_{2}$, as evidenced by its well-retained crystallinity, morphology and surface area after ageing under the aforementioned conditions for 7 days (Fig. 6e-h). In sharp contrast, as observed from PXRD patterns, MOF-5 starts to transform to the non-porous MOF-69 under the humidified $\mathrm{CO}_{2}$ environment within $<1.5 \mathrm{~h}$ and such a transformation almost 
finishes after $4 \mathrm{~h}$ as indicated by the complete disappearance of MOF-5 phase in the PXRD patterns. Correspondingly, the SEM images show that the MOF- 5 crystals underwent serious corrosion along with the complete loss of its porosity after $4 \mathrm{~h}$ (Fig. 6a-d). These results highlight the chemical shielding effect of amphiphobicity in protecting the highly unstable MOF-5 analogues under various conditions such as humidified $\mathrm{CO}_{2}$ atmosphere, environment with high humidity and aqueous solutions (Supplementary Figs 25-27). The greatly enhanced stability of amphiphobic MOFs over their pristine ones in both acidic and basic aqueous solutions further underscore the chemical shielding effect of amphiphobicity (Supplementary Figs 28-37).

In summary, we have demonstrated the successful impartment of amphiphobicity (that is, superhydrophobic and oleophobic) on the exterior surface of the highly single-crystalline porous materials of MOFs. Such amphiphobic surface can serve as a chemical shield to effectively prevent the MOFs from being attacked by water and organic compounds, thereby bestowing the MOFs with ultrastability towards moisture/water and humidified $\mathrm{CO}_{2}$. Our approach contributed herein to create amphiphobic surface has little impact on the crystallinity and porosity of the pristine MOF materials, thereby pioneering a perspective to protect crystalline porous materials under various chemical environments for numerous applications.

\section{Methods}

Materials and measurements. Commercially available reagents were purchased in high purity and used without purification. Solvents were purified according to standard laboratory methods. Tetrahydrofuran (THF) was distilled over $\mathrm{LiAlH}_{4}$. Dimethylformamide (DMF) was distilled over $\mathrm{CaH}_{2}$. Nitrogen sorption isotherms at the temperature of liquid nitrogen were measured using Micromeritics ASAP $2020 \mathrm{M}$ and Tristar System. The samples were outgassed for $12 \mathrm{~h}$ at $100^{\circ} \mathrm{C}$ before the measurements. ${ }^{1} \mathrm{H}$ NMR spectra were recorded on a Bruker Avance- 400 $(400 \mathrm{MHz})$ spectrometer. Chemical shifts are expressed in p.p.m. downfield from tetramethylsilane (TMS) at $\delta=0$ p.p.m. and $J$ values are given in Hz. SEM was performed on a Hitachi SU 1510. PXRD patterns were measured with a Rigaku Ultimate VI X-ray diffractometer $(40 \mathrm{kV}, 40 \mathrm{~mA})$ using $\mathrm{CuK} \alpha(\lambda=1.5406 \AA)$ radiation. Photographs of water and organic compounds on the surface of the samples in the pressed pellet form were measured with SL200KB (USA KNO Industry, Co.), equipped with a charge-coupled device camera.

$\mathrm{X}$-ray photoemission spectra were performed on a Thermo ESCALAB 250 with $\mathrm{Al} \mathrm{K} \alpha$ irradiation at $\theta=90^{\circ}$ for X-ray sources and the binding energies were calibrated using the $\mathrm{C} 1 \mathrm{~s}$ peak at $284.9 \mathrm{eV}$. $\mathrm{An} \mathrm{Ar}^{+}$sputter beam ( $2 \mathrm{keV}, 100 \mathrm{~s}$ ) was used for depth profiling of ZIF-8-VF and MOF-5-VF after the initial data was collected. High-angle annular dark field scanning, scanning transmission electron microscopic imaging and energy-dispersive X-ray spectroscopy mapping were carried out by Titan ChemiSTEM operated at $200 \mathrm{kV}$. Water adsorption and desorption isotherms were obtained via SMS Instruments DVS Advantage. The balance has a sensitivity of $0.1 \mu \mathrm{g}$. These isotherms were measured at $25^{\circ} \mathrm{C}$ by monitoring the weight change of the sample as a function of relative humidity of water. The relative humidity of water was stepped up from 0 to $98 \%$ with an increment of $10 \%$ in each step and then was stepped down to $0 \%$. Real-time weight, temperature and relative humidity were recorded. Toluene adsorption isotherms were measured via Micromeritics 3Flex. These isotherms were collected at $25^{\circ} \mathrm{C}$ by monitoring the volume change.

Synthesis of single-crystal ZIF-8-V. Single crystal of ZIF-8-V was obtained by slowly evaporating the mixture of trimethylamine $(3 \mu \mathrm{l})$ and cyclohexane into a DMF $(2 \mathrm{ml})$ solution of $\mathrm{Zn}\left(\mathrm{NO}_{3}\right)_{2} \cdot 6 \mathrm{H}_{2} \mathrm{O}(0.033 \mathrm{mmol})$ and 2-vinyl-imidazole $(0.1 \mathrm{mmol})$ for 7 days at room temperature.

Synthesis of powder ZIF-8-V crystal. 2-Vinyl-imidazole $(0.094 \mathrm{~g}, 1.0 \mathrm{mmol})$ and zinc nitrate hexahydrate $\left[\mathrm{Zn}\left(\mathrm{NO}_{3}\right)_{2} \cdot 6 \mathrm{H}_{2} \mathrm{O}\right](0.149 \mathrm{~g}, 0.50 \mathrm{mmol})$ in DMF $(15 \mathrm{ml})$ was placed in a desiccator under an atmosphere of the mixture of triethylamine $(5 \mathrm{ml})$ and cyclohexane $(200 \mathrm{ml})$. The reaction was allowed to proceed at room temperature for $48 \mathrm{~h}$. The crystalline powder was obtained by centrifugation, washed with methanol $(3 \times 25 \mathrm{ml})$ and activated with methanol $(3 \times 25 \mathrm{ml})$ for 3 days before being dried under vacuum at room temperature. Yield $(0.09 \mathrm{~g}, 72 \%) \mathrm{CHN}$ calculated for $\mathrm{C}_{10} \mathrm{H}_{10} \mathrm{~N}_{4} \mathrm{Zn}$ : C, 48.0; H, 4.0; N, 22.3\%. Found: C, $48.3 ; \mathrm{H}, 4.40 ; \mathrm{N}, 20.1 \%$.
Covalent post-synthetic modification of ZIF-8-V. Activated ZIF-8-V powder $(0.10 \mathrm{~g})$ was suspended in (trifluoromethyl)benzene $(10 \mathrm{ml})$ solution containing $10 \mathrm{v} / \mathrm{v} \% 1 \mathrm{H}, 1 \mathrm{H}, 2 \mathrm{H}, 2 \mathrm{H}$-perfluorodecanethiol and catalytic amount of azobisisobutyronitrile. The reaction was carried out at $60^{\circ} \mathrm{C}$ for $10 \mathrm{~h}$ under $\mathrm{N}_{2}$, to attach perfluoroalkyl groups on the crystal surface by the thiol-ene click reaction. The product denoted as ZIF-8-VF was obtained by centrifugation, washed with methanol $(3 \times 25 \mathrm{ml})$ and dried under vacuum at room temperature. $\mathrm{CHN}$ found for ZIF-8-VF: C, 49.3; H, 4.49; N, 18.5\%.

Data availability. The authors declare that the data supporting the findings of this study are available within the article and its Supplementary Information files and from the corresponding author upon reasonable request.

\section{References}

1. Liu, M., Zheng, Y., Zhai, J. \& Jiang, L. Bioinspired super-antiwetting interfaces with special liquid-solid adhesion. Acc. Chem. Res. 43, 368-377 (2010).

2. Darmanin, T., Taffin de Givenchy, E., Amigoni, S. \& Guittard, F. Superhydrophobic surfaces by electrochemical processes. Adv. Mater. 25, 1378-1394 (2013).

3. Li, X.-M., Reinhoudt, D. \& Crego-Calama, M. What do we need for a superhydrophobic surface? A review on the recent progress in the preparation of superhydrophobic surfaces. Chem. Soc. Rev. 36, 1350-1368 (2007).

4. Zhang, C., Mcadams, D. A. II. \& Grunlan, J. C. Nano/micro-manufacturing of bioinspired materials: a review of methods to mimic natural structures. Adv. Mater. 28, 6292-6321 (2016).

5. Su, B., Tian, Y. \& Jiang, L. Bioinspired interfaces with superwettability: from materials to chemistry. J. Am. Chem. Soc. 138, 1727-1748 (2016).

6. Xu, Q. et al. Robust self-cleaning and micromanipulation capabilities of gecko spatulae and their bio-mimics. Nat. Commun. 6, 8949 (2015)

7. Huang, G., Yang, Q., Xu, Q., Yu, S.-H. \& Jiang, H.-L. Polydimethylsiloxane coating for a palladium/MOF composite: highly improved catalytic performance by surface hydrophobization. Angew. Chem. Int. Ed. 55, 7379-7383 (2016).

8. Bellanger, H., Darmanin, T., Taffin de Givenchy, E. \& Guittard, F. Chemical and physical pathways for the preparation of superoleophobic surfaces and related wetting theories. Chem. Rev. 114, 2694-2716 (2014).

9. Pan, S., Kota, A. K., Mabry, J. M. \& Tuteja, A. Superomniphobic surfaces for effective chemical shielding. J. Am. Chem. Soc. 135, 578-581 (2013).

10. Tuteja, A. et al. Designing superoleophobic surfaces. Science 318, 1618-1622 (2007).

11. Kota, A. K., Li, Y., Mabry, J. M. \& Tuteja, A. Hierarchically structured superoleophobic surfaces with ultralow contact angle hysteresis. Adv. Mater. 21, 665-669 (2009).

12. Liu, M., Wang, S., Wei, Z., Song, Y. \& Jiang, L. Bioinspired design of a superoleophobic and low adhesive water/solid interface. Adv. Mater. 21, 665-669 (2009)

13. Xue, Z. et al. A novel superhydrophilic and underwater superoleophobic hydrogel-coated mesh for oil/water separation. Adv. Mater. 23, 4270-4273 (2011).

14. Ionov, L., Houbenov, N., Sidorenko, A., Stamm, M. \& Minko, S. Smart microfluidic channels. Adv. Funct. Mater. 16, 1153-1160 (2006).

15. Xu, Z., Zhao, Y., Wang, H., Wang, X. \& Lin, T. A superamphiphobic coating with an ammonia-triggered transition to superhydrophilic and superoleophobic for oil-water separation. Angew. Chem. Int. Ed. 54, 4527-4530 (2015).

16. Zhou, H.-C. \& Kitagawa, S. Metal-organic frameworks (MOFs). Chem. Soc. Rev 43, 5415-5418 (2014).

17. James, S. L. Metal-organic frameworks. Chem. Soc. Rev. 32, 276-288 (2003).

18. Furukawa, H., Cordova, K. H., O'Keeffe, M. \& Yaghi, O. M. The chemistry and applications of metal-organic frameworks. Science 341, 1230444 (2013).

19. Ma, S. \& Zhou, H.-C. Gas storage in porous metal-organic frameworks for clean energy applications. Chem. Commun. 46, 44-53 (2010).

20. Suh, M. P., Park, H. J., Prasad, T. K. \& Lim, D.-W. Hydrogen storage in metal-organic frameworks. Chem. Rev. 112, 782-835 (2011).

21. Hu, T.-L. et al. Microporous metal-organic framework with dual functionalities for highly efficient removal of acetylene from ethylene/acetylene mixtures. Nat. Commun. 6, 7328 (2015).

22. Liao, P.-Q., Zhang, W.-X., Zhang, J.-P. \& Chen, X.-M. Efficient purification of ethene by an ethane-trapping metal-organic framework. Nat. Commun. 6, 8697 (2015).

23. McDonald, T. M. et al. Cooperative insertion of $\mathrm{CO}_{2}$ in diamineappended metal-organic frameworks. Nature 519, 303-308 (2015).

24. Rabone, J. et al. An adaptable peptide-based porous material. Science 329, 1053-1057 (2010).

25. Liu, J. et al. Applications of metal-organic frameworks in heterogeneous supramolecular catalysis. Chem. Soc. Rev. 43, 6011-6061 (2014).

26. Saha, S., Das, G., Thote, J. \& Banerjee, R. Photocatalytic metal-organic framework from CdS quantum dot incubated luminescent metallohydrogel. J. Am. Chem. Soc. 136, 14845-14851 (2014). 
27. Mo, K., Yang, Y. \& Cui, Y. A homochiral metal-organic framework as an effective asymmetric catalyst for cyanohydrin synthesis. J. Am. Chem. Soc. 136, 1746-1749 (2014).

28. Li, Z. et al. Platinum-nickel frame within metal-organic framework fabricated in situ for hydrogen enrichment and molecular sieving. Nat. Commun. 6, 8248 (2015).

29. Zhou, J. et al. A bimetallic lanthanide metal-organic material as a self-calibrating color-gradient luminescent sensor. Adv. Mater. 27, 7072-7077 (2015).

30. Hu, Z., Deibert, B. J. \& Li, J. Luminescent metal-organic frameworks for chemical sensing and explosive detection. Chem. Soc. Rev. 43, 5815-5840 (2014).

31. Peng, Y. et al. Engineering chiral porous metal-organic frameworks for enantioselective adsorption and separation. Nat. Commun. 5, 4406 (2014).

32. Van de Voorde, B., Bueken, B., Denayer, J. \& De Vos, D. Adsorptive separation on metal-organic frameworks in the liquid phase. Chem. Soc. Rev. 43, 5766-5788 (2014).

33. Stavila, V., Talin, A. A. \& Allendorf, M. D. MOF-based electronic and opto-electronic devices. Chem. Soc. Rev. 43, 5994-6010 (2014).

34. Park, S. S. et al. Cation-dependent intrinsic electrical conductivity in isostructural tetrathiafulvalene-based microporous metal-organic frameworks. J. Am. Chem. Soc. 137, 1774-1777 (2015).

35. Yao, J. \& Wang, H. Zeolitic imidazolate framework composite membranes and thin films: synthesis and applications. Chem. Soc. Rev. 43, 4470-4493 (2014).

36. Pera-Titus, M. Porous inorganic membranes for $\mathrm{CO}_{2}$ capture: present and prospects. Chem. Rev. 114, 1413-1492 (2014).

37. DeCoste, J. B. \& Peterson, G. W. Metal-organic frameworks for air purification of toxic chemicals. Chem. Rev. 114, 5695-5727 (2014).

38. Burtch, N. C., Jasuja, H. \& Walton, K. S. Water stability and adsorption in metal-organic frameworks. Chem. Rev. 114, 10575-10612 (2014).

39. Mottillo, C. \& Friščić, T. Carbon dioxide sensitivity of zeolitic imidazolate frameworks. Angew. Chem. Int. Ed. 53, 7471-7474 (2014).

40. Liu, H. et al. Irreversible change of the pore structure of ZIF-8 in carbon dioxide capture with water coexistence. J. Phys. Chem. C 120, 13287-13294 (2016).

41. Decoste, J. B., Peterson, G. W., Smith, M. W., Stone, C. A. \& Willis, C. R. Enhanced stability of Cu-BTC MOF via perfluorohexane plasma-enhanced chemical vapor deposition. J. Am. Chem. Soc. 134, 1486-1489 (2012).

42. Carné-Sánchez, A. et al. Protecting metal-organic framework crystals from hydrolytic degradation by spray-dry encapsulating them into polystyrene microspheres. Adv. Mater. 27, 869-873 (2015).

43. Tanabe, K. K. \& Cohen, S. M. Postsynthetic modification of metal-organic frameworks-a progress report. Chem. Soc. Rev. 40, 498-519 (2011).

44. Nguyen, J. G. \& Cohen, S. M. Moisture-resistant and superhydrophobic metalorganic frameworks obtained via postsynthetic modification. J. Am. Chem. Soc. 132, 4560-4561 (2010).

45. McGuire, C. V. \& Forgan, R. S. The surface chemistry of metal-organic frameworks. Chem. Commun. 51, 5199-5217 (2015).

46. Yang, S. J. \& Park, C. R. Preparation of highly moisture-resistant black-colored metal organic frameworks. Adv. Mater. 24, 4010-4013 (2012).

47. Jayaramulu, K. et al. Biomimetic superhydrophobic/superoleophilic highly fluorinated graphene oxide and ZIF-8 composites for oil-water separation. Angew. Chem. Int. Ed. 55, 1178-1182 (2016).

48. Deria, P. et al. Perfluoroalkane functionalization of NU-1000 via solventassisted ligand incorporation: synthesis and $\mathrm{CO}_{2}$ adsorption studies. J. Am. Chem. Soc. 135, 16801-16804 (2013).

49. Rao, K. P. et al. Design of superhydrophobic porous coordination polymers through the introduction of external surface corrugation by the use of an aromatic hydrocarbon building unit. Angew. Chem. Int. Ed. 53, 7471-7474 (2014).

50. Chen, T.-H. et al. Thermally robust and porous noncovalent organic framework with high affinity for fluorocarbons and CFCs. Nat. Commun. 5, 5131 (2014).

51. Chen, T.-H., Popov, I., Zenasni, O., Daugulis, O. \& Miljanić, O. Š. Superhydrophobic perfluorinated metal-organic frameworks. Chem. Commun. 49, 6846-6848 (2013)

52. Chen, T.-H. et al. Mesoporous fluorinated metal-organic frameworks with exceptional adsorption of fluorocarbons and CFCs. Angew. Chem. Int. Ed. 54, 13902-13906 (2015).

53. Zhang, Z. et al. Polymer-metal-organic frameworks (polyMOFs) as water tolerant materials for selective carbon dioxide separations. J. Am. Chem. Soc. 138, 920-925 (2016).
54. Zhang, W., Hu, Y., Ge, J., Jiang, H.-L. \& Yu, S.-H. A facile and general coating approach to moisture/water-resistant metal-organic frameworks with intact porosity. J. Am. Chem. Soc. 136, 16978-16981 (2014).

55. Wang, Y. Q. et al. Directing the formation of vinyl-functionalized silica to the hexagonal SBA-15 or large-pore Ia3d structure. Chem. Mater. 15, 5029-5035 (2003).

56. Morris, W., Doonan, C. J., Furukawa, H., Banerjee, R. \& Yaghi, O. M. Crystals as molecules: postsynthesis covalent functionalization of zeolitic imidazolate frameworks. J. Am. Chem. Soc. 130, 12626-12627 (2008).

57. Hayase, G. et al. A superamphiphobic macroporous silicone monolith with marshmallow-like flexibility. Angew. Chem. Int. Ed. 52, 10788-10791 (2013).

58. Serre, C. Superhydrophobicity in highly fluorinated porous metal-organic frameworks. Angew. Chem. Int. Ed. 51, 6048-6051 (2012).

59. Yang, C. et al. Fluorous metal-organic frameworks with superior adsorption and hydrophobic properties toward oil spill cleanup and hydrocarbon storage. J. Am. Chem. Soc. 133, 18094-18097 (2011).

60. Padial, N. M. et al. Highly hydrophobic isoreticular porous metal-organic frameworks for the capture of harmful volatile organic compounds. Angew. Chem. Int. Ed. 52, 8290-8294 (2013).

61. Zhang, J.-P., Zhang, Y.-B., Lin, J.-B. \& Chen, X.-M. Metal azolate frameworks from crystal engineering to functional materials. Chem. Rev. 112, 1001-1033 (2012).

62. Phan, A. et al. Synthesis, structure, and carbon dioxide capture properties of zeolitic imidazolate frameworks. Acc. Chem. Res. 43, 58-67 (2010).

\section{Acknowledgements}

This work was supported by the National Natural Science Foundation of China (21273197, 21333009 and 21422306) and National High-Tech Research and Development programme of China (2013AA065301). Financial support from the University of South Florida and the National Science Foundation (DMR-1352065) is also acknowledged (S.M.). The single-crystal XRD of MOF-5-V was carried out at the Advanced Photo Source on beamline 15-ID-B of ChemMatCARS (NSF/CHE-0822838 and DE-AC02-06CH11357). ChemMatCARS Sector 15 is supported by the National Science Foundation under grant number NSF/CHE-1346572. This research used resources of the Advanced Photon Source, a U.S. Department of Energy (DOE) Office of Science User Facility operated for the DOE Office of Science by Argonne National Laboratory under Contract Number DE-AC02-06CH11357.

\section{Author contributions}

Q.S., F.-S.X. and S.M. conceived and designed the research. Q.S., B.A. and S.M. drafted the manuscript. Q.S. and H.H. carried out the synthesis. W.-Y.G., L.W. and Y.-S.C. collected and analysed the single-crystal XRD data. Q.S. conducted the NMR characterization of the materials. Z.D. carried out the contact angle tests. J.L. contributed to the transmission electron microscopy tests. All authors discussed the results and gave approval to the final version of the manuscript.

\section{Additional information}

Supplementary Information accompanies this paper at http://www.nature.com/ naturecommunications

Competing financial interests: The authors declare no competing financial interests

Reprints and permission information is available online at http://npg.nature.com/ reprintsandpermissions/

How to cite this article: Sun, Q. et al. Imparting amphiphobicity on single-crystalline porous materials. Nat. Commun. 7, 13300 doi: 10.1038/ncomms13300 (2016).

Publisher's note: Springer Nature remains neutral with regard to jurisdictional claims in published maps and institutional affiliations.

This work is licensed under a Creative Commons Attribution 4.0 International License. The images or other third party material in this article are included in the article's Creative Commons license, unless indicated otherwise in the credit line; if the material is not included under the Creative Commons license, users will need to obtain permission from the license holder to reproduce the material. To view a copy of this license, visit http://creativecommons.org/licenses/by/4.0/

(C) The Author(s) 2016 